\title{
Real-Time Automatic Defect Inspection of PCTP ITO Circuits Using a 1-D Image Comparison Algorithm
}

\author{
Changcheng Jiang ${ }^{1}$, Yanming Quan ${ }^{1 *}$ and Xingui Lin $^{2}$ \\ ${ }^{1}$ School of Mechanical and Automotive Engineering, South China University of \\ Technology, Guangzhou, China \\ ${ }^{2}$ School of Electro mechanic Engineering, Guangzhou Panyu Polytechnic, \\ Guangzhou, China \\ changchengjiang@qq.com,meymquan@scut.edu.cn,linxg@gzpyp.edu.cn
}

\begin{abstract}
Touch panels have gained wide popularity in many consumer electronics such as smartphones, laptops and tablets. This paper focuses on the issue of automatic visual inspection of defects in Indium Tin Oxide (ITO) circuits on the surfaces of projective capacitive touch panel (PCTP). We propose a fast defect detection algorithm that directly compares the cross-pixels of one-dimensional (1-D) images between sensed images and template images. In our method, a faultless template image is extracted from three sensed images first. Then, we compare the number and coordinate differences of the cross-pixels between the sensed image and template image to identify defects and their location. Experiment results show that the proposed method can quickly and accurately detect various defects in the surface of PCTP ITO circuits. It also passes the robustness check under different illumination conditions. Therefore, this algorithm can be reliably used in real-time inspection of PCTP ITO circuits.
\end{abstract}

Keywords: Projective capacitive touch panel (PCTP), Indium Tin Oxide (ITO) circuits, Defect detection, 1-D image processing, Line scan

\section{Introduction}

Touch panels (TPs, also named touchscreens) are widely used in nowaday computers, tablets, smartphones, etc., as an input device [1]. Among all types of TPs, PCTP has become the dominant type due to its high resolution, good light transmission, multi-touch and low cost. In the PCTP manufacturing process, the most important procedure is to form patterned ITO circuits on both sides of a substrate using wet etching [2], plasma etching [3] or laser ablation [4]. However, all current etching methods will inevitably leave defects on PCTP ITO circuits, including pinholes, scratches, particles, short circuits and open circuits. It is crucial to detect and repair these defects as they often cause the whole TP to fail.

With the rapid development of microfabrication technology, the etching density of PCTP ITO circuits has increased from tens of microns to a few microns in width. Correspondingly, defects have become smaller and smaller and cannot be easily detected by visual inspection. To ensure the quality and quantity production of PCTPs, the defect inspection of ITO circuits becomes a critical task in PCTP manufacturing. Many automatic detection methods have been developed. Electric test is one of them. Chan et. al., presented an electrical inspection system for ITO circuits [5]. In the system, a polymer dispersed liquid crystal (PDLC) film is used as a sensing device to locate faulty circuits. The ITO circuits and PDLC film are both linked to an external power source to form an electric field. With the power on, the crystals in the conducting area are different from

${ }^{*}$ Corresponding Author 
those in the non-conducting area. Those differences can be imaged by a camera and the defect location can be identified in the image. However, the main disadvantages of such electric test approaches are as follows. They can only inspect short and open circuits, and are becoming less and less effective in inspecting ITO circuits with increasing density.

Machine vision technique has also been investigated for defect detection [6-8], and many researches on TFT-LCD and IC circuits have been published. Hou et al. proposed a pattern matching method based on mathematical morphology [9]. In their method, the differences between the sensed image and the template image are first converted into a binary image, which is then analyzed using mathematical morphology to detect and classify the defects. This approach is based on two-dimensional (2-D) grayscale images. The sensed images cannot be deformed from template image in order for the method to work properly. Tsai and Hung proposed a global 1-D line Fourier-based image reconstruction algorithm for TFT-LCD defect detection at a coarse resolution of 60 pixels/mm [10]. In their method, the sensed image is first decomposed into 1-D line images, which are then transformed from the Time domain to the Fourier domain. The frequency components that represent the periodic pattern of TFT-LCD lines are then eliminated from the Fourier domain so that local anomalies are preserved in the resulting 1-D line image. Wavelet decomposition is further applied to remove uneven illumination. The approach is applicable only to surfaces with uniformed and repeated regularity. Tsai and Lai used independent component analysis (ICA) for TFT-LCD defect detection at a fine resolution of 100 pixels $/ \mathrm{mm}$ [11]. The sensed 1-D line image is first divided into two segments of equal length. Then the de-mixing matrix from a faultless line image is used to recover the signals with well aligned translation. The normalized cross-correlation is finally adopted to measure the similarity between the two segments to locate defects. The defects can be further classified by comparing the sensed images with normal patterns. However, the main disadvantage of this method is that it is time-consuming, especially when processing huge amount of data. Therefore, it cannot be used in real-time inspection. Liu et. al., proposed a frequency transformation method and a 2-D Wavelet transformation (WT) method to inspect defects in the Integrated Circuit (IC) image [1213]. In their methods, three images with defects located in the same position of different chips are processed using spectral subtraction or 2-D WT to get a faultless template image first. Then, sensed images are compared with the template image to locate defects. These two methods are not applicable to real inspection for the following reasons. First, sensed images can be deformed which affects inspection accuracy. Second, they require the gray values of the corresponding locations in the two images to be the same, which is impossible in real inspection.

Currently, researches on ITO patterns or circuits inspection based on machine vision technique are sparse. Song et. al., proposed a threshold segmentation method for defect inspection of PDP ITO pattern [14]. Their method is based on the fact that ITO patterns and substrates represent two different peaks in the histogram of the sensed images. Therefore, one can use threshold segmentation to remove the gray values corresponding to the ITO patterns, and what is left in the sensed image is just the defect. This method is simple and fast. But in real inspection, the ideal sensed image is difficult to obtain due to environmental noises, image deformation and uneven illumination. These will inevitably cause partial overlap between the two peaks in the histogram. Therefore, this method will often lead to wrong conclusions when used in real inspection. Liu and Peng presented a template reconstruction method for defect detection of ITO patterns in LCD TP [15]. The core idea of their method is based on the fact that the structure of ITO patterns in LCD TP is simple and they have fixed repeated cycles. A faultless template can then be established by calculating the shape and periodicity in sensed images. Subtracting the template from the sensed image identifies the defect. This method is also simple and fast. However, it is only applicable to area-scan automated optical inspection (AOI) system. It also requires 
sensed images to be minimally deformed and the ITO patterns to be of simple regular shape such as rectangle, rhombus, etc.,.

With the increasing etching density of ITO circuits, high detection accuracy and realtime processing and display become essential. Consequently, the AOI system can only be established based on one or more high-resolution line scans in real inspection. Moreover, since the line-scan AOI system inevitably leads to a certain degree of mechanical vibration and uneven illumination, it can cause deformation in sensed images. Therefore, the detection algorithms mentioned above do not meet the needs of real-time line-scan inspection of PTCP ITO circuits.

In this study, we propose an AOI algorithm that identifies defects directly from 1-D images of PCTP ITO circuits at a fine resolution of 100 pixels $/ \mathrm{mm}$. A faultless template is extracted from sensed images first, and defects can then be easily found by comparing gray value distributions of the 1-D images between sensed images and the template image. Thus, our method does not rely on the combination of multiple complex image processing algorithms or mathematical transformations to describe local anomalies. It saves time and has high inspection accuracy.

\section{Theory of Defect Detection for PCTP ITO Circuits Based on 1-D Images}

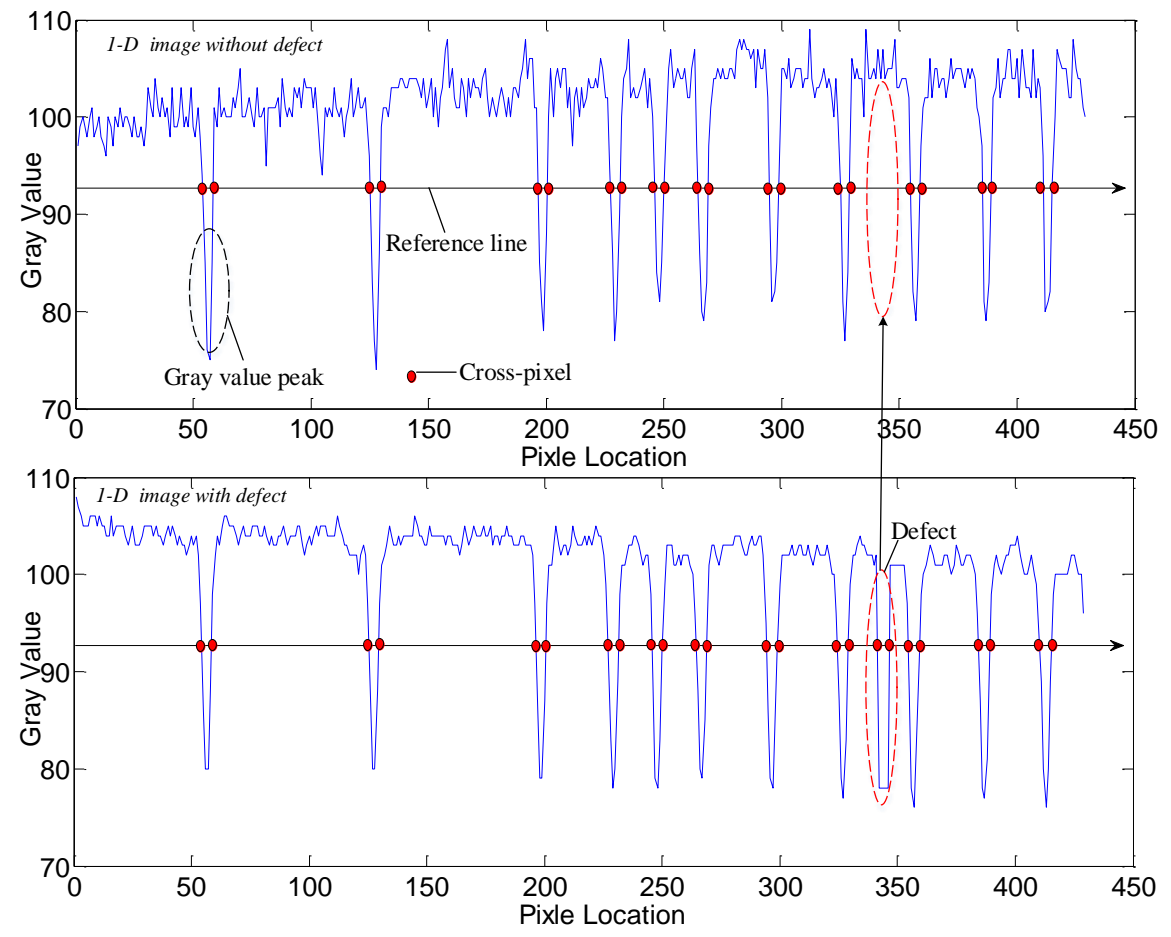

Figure 1. Principle of Defect Detection by Comparing 1-D Images

Suppose a sensed grayscale image is of size $M \times N$ pixels. Decompose it by row into $M$ row matrixes. Each row matrix represents the gray value distribution of the $N$ pixels in this row. We name such row matrix 1-D image. In the 1-D images of PCTP ITO circuits, even if sensed images are deformed, the number of gray value peaks, which is determined by the change from low gray values (corresponding to the substrate) to high gray values (corresponding to ITO circuits) and the reverse change, is invariable. Therefore, whether the sensed 1-D images have defects can be easily recognized by comparing the number of gray value peaks in the template with the number in the sensed 1-D images. In order to accurately obtain the gray value peaks in a 1-D image, we first draw a reference line whose value is equal to the threshold of the 1-D image. The reference line intersects every 
gray value peak at two pixels, and these pixels are named cross-pixels. So the number of intersections is twice the number of gray value peaks. Furthermore, the defect position can be found by comparing the coordinate differences of all the cross-pixels between sensed and template 1-D line image (as shown in Figure 1).

To use our method, a faultless template image needs to be obtained first. However, in real inspection of PCTP ITO circuits, uneven illumination, environmental influences, lens distortion and mechanical vibration caused by the relative movements between the linescans and the inspection object inevitably lead to deformation of the sensed images. If the ideal template image is directly derived from DXF files, although the number of crosspixels in every 1-D image is still the same, their coordinates often mismatch those in the sensed images due to deformation in the sensed images. This will result in wrong detection conclusions. Therefore, the template image needs to be extracted from sensed images so that they are under the same imaging conditions and can be used for real inspection. In what follows, we propose a two-step defect detection process: extraction of a faultless template image and defect detection using the template.

\subsection{Extracting a Faultless Template}

2.1.1. Determining the Number of Reference Images In PCTP manufacturing, one substrate holds multiple PCTPs with identical ITO circuits. During inspection, several identical ITO circuits are scanned into one image at once. For example, Figure 2, shows the structure of a typical PCTP substrate. There are 18 identical ITO circuits distributed in 3 rows. If the substrate moves in the direction as shown in Figure 2 (a), 6 identical ITOs are imaged at once.

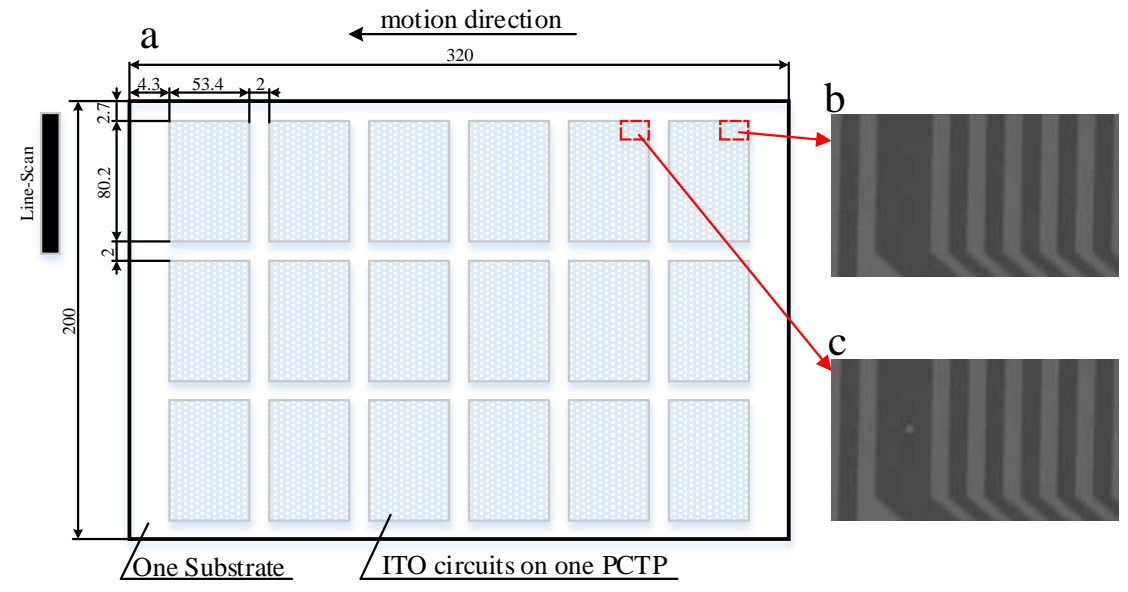

Figure 2. (a) Typical Structure of a PCTP Substrate and Inspection Scheme; (b) Partial Image of an ITO Circuit without Defect; (c) Partial Image of an ITO Circuit with a Pinhole

After scanning, we first extract the image of each ITO circuit. Each image is of size $M \times N$ pixels. Suppose the defect probability in each PCTP ITO circuit is $\xi$. In real inspection, defect areas that are greater than $n_{3}$ pixels need to be identified. Thus, the probability that a defect exists in the same location of $n$ ITO circuit images, $P$, can be calculated as

$P=\left(\frac{\xi n_{3}}{M N}\right)^{n}$

In real inspection, the probability that a defect exists in the same location of two ITO circuit images is very low. It is almost zero for three images. To illustrate this point, let's take a 4" PCTP ITO circuit as an example. Its size $M \times N=8854 \times 4980$ pixels under a fine 
resolution of 100 pixels $/ \mathrm{mm} . n_{3}$ is usually 5 pixels under such resolution. Suppose $\xi$ is $10 \%$. Then, when $n=3, P=1.45 \times 10^{-24} \approx 0$. As the size of PCTP increases, $P$ decreases exponentially. Therefore, using three sensed images (named reference images) to extract a faultless template is appropriate.

2.1.2. 1-D Image Decomposition and Its Reference Line: Denote by $P_{1}, P_{2}$ and $P_{3}$ three reference images that may have defects. Their sizes are all $M \times N$ pixels. After decomposition by row, $F_{i}=\left(f_{(i, 0)}, f_{(i, 1)}, \ldots, f_{(i, M-1)}\right), G_{i}=\left(g_{(i, 0)}, g_{(i, 1)}, \ldots, g_{(i, M-1)}\right)$ and $H_{i}=\left(h_{(i, 0)}\right.$, $\left.h_{(i, 1)}, \ldots, h_{(i, M-1)}\right)$ represent the 1-D image of the $\mathrm{i}^{\text {th }}$ row of $\mathrm{P}_{1}, \mathrm{P}_{2}$ and $\mathrm{P}_{3}$, respectively $(i \epsilon(0, N$ 1)). For example, Figure 3, shows $\mathrm{P}_{1}, \mathrm{P}_{2}$ and $\mathrm{P}_{3}$, and the 1-D images of their $28^{\text {th }}$ row $F_{28}$, $G_{28}$ and $H_{28}$.

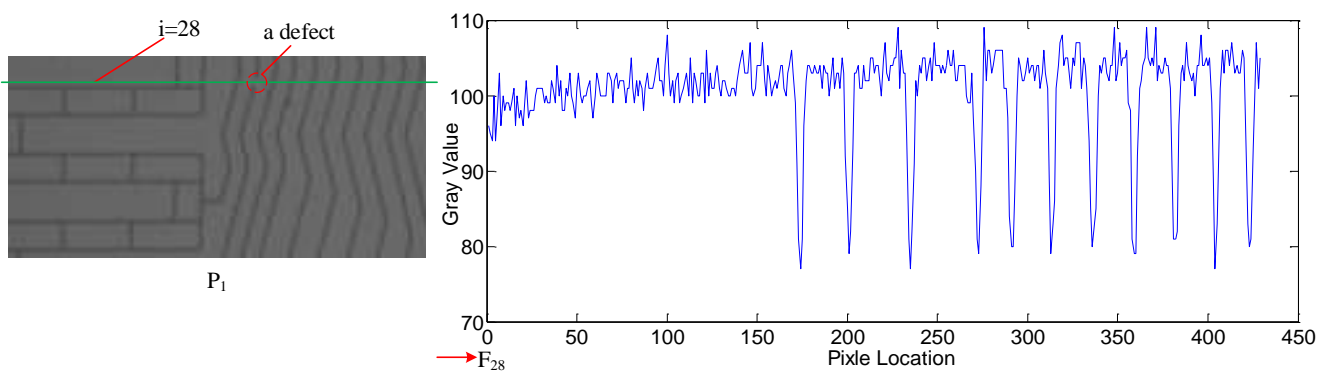

(a) $\mathrm{P}_{1}$ (the left figure) and 1-D image $F_{28}$ (the right figure)

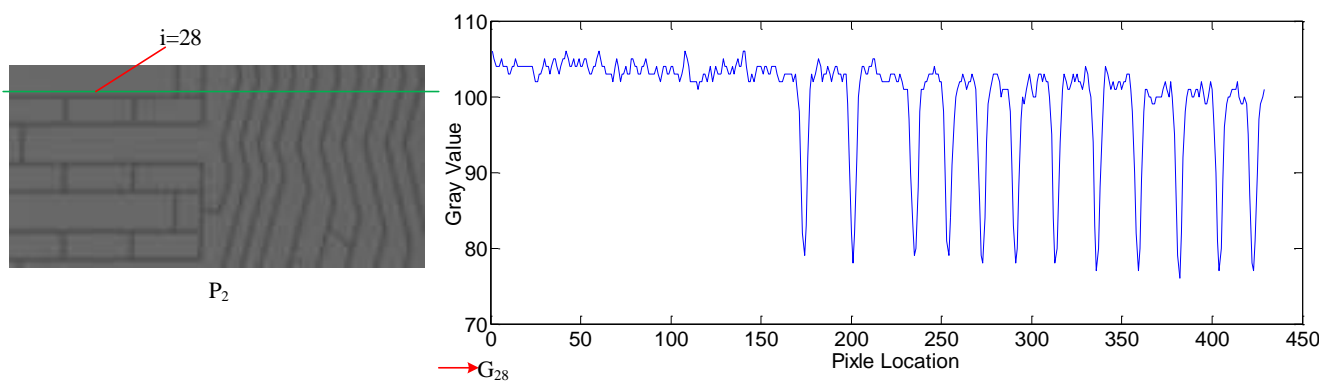

(b) $P_{2}$ (the left figure) and 1-D image $G_{28}$ (the right figure)

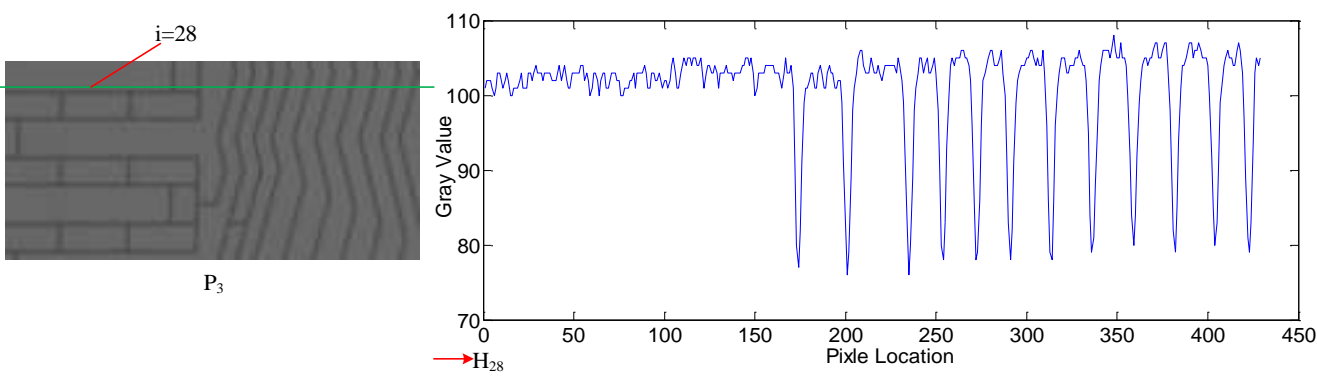

(c) $P_{3}$ (the left figure) and 1-D image $H_{28}$ (the right figure)

Figure 3. Three Reference Images and Their 1-D Images When $i=28$

In the 1-D image of a PCTP ITO circuit, the gray value distribution of all its pixels, $g_{(i, j)}$, usually contains $g_{l(i, j)}$, a set of pixels with low gray values (corresponding to the ITO circuit) and $g_{h(i, j)}$, a set of pixels with high gray values (corresponding to the substrate). And its statistical distribution can be roughly approximated combining two Normal distributions:

$$
g_{(i, j)} \approx g_{l(i, j)}+g_{h(i, j)}
$$




$$
\left\{\begin{array}{l}
g_{l(i, j)} \sim N_{1}\left(\mu_{1}, \sigma_{1}^{2}\right)=\frac{1}{\sqrt{2 \pi} \sigma_{1}} e^{-\frac{\left(g_{l(i, j)}-\mu_{1}\right)^{2}}{2 \sigma_{1}^{2}}} \\
g_{h(i, j)} \sim N_{2}\left(\mu_{2}, \sigma_{2}^{2}\right)=\frac{1}{\sqrt{2 \pi} \sigma_{2}} e^{-\frac{\left(g_{h(i, j)}-\mu_{2}\right)^{2}}{2 \sigma_{2}^{2}}}
\end{array}\right.
$$

where $\mu_{1}$ and $\mu_{2}$ represent the average gray value of the two sets, respectively.

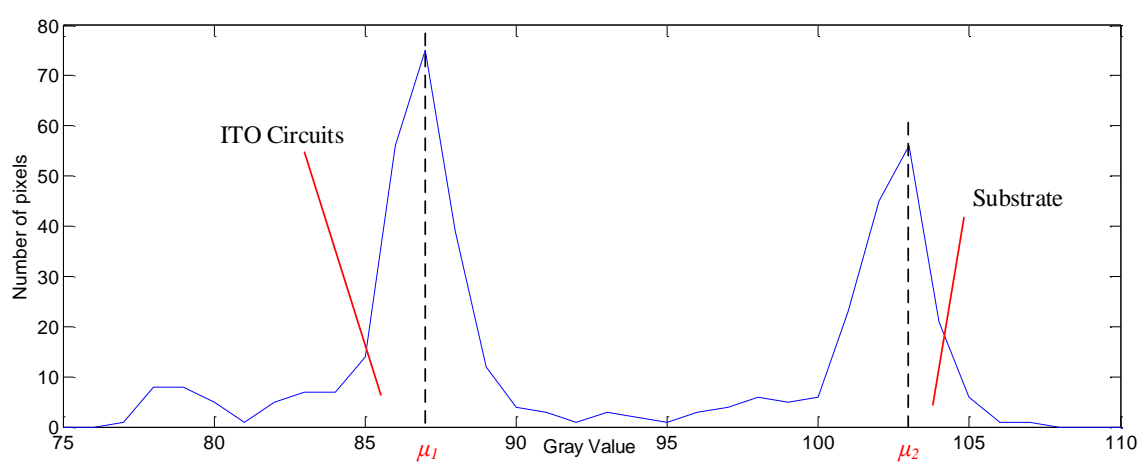

Figure 4. Histogram of 1-D Images $\left(G_{28}\right)$

The key part of our algorithm is to set the reference line in each 1-D image in a way that it helps accurately locate the cross-pixels. To achieve that goal, the reference line needs to be set across the transition area between high and low gray value's peaks in the histogram and be away from $\mu_{1}$ and $\mu_{2}$ (as shown in Figure 4). Moreover, for different $i$, the gray value distribution of its 1-D image can be very different. So setting a fixed reference line for all i's is not appropriate. We choose to set the reference line at the threshold value of each 1-D image. And in order to speed up the process, we utilize the automatic threshold segmentation algorithm to calculate the thresholds [16].

2.1.3. Defect Detection in the Target Reference Image: Extracting cross-pixels is fundamental to locating defects in 1-D images. By calculating three new 1-D images $\left(F_{\varepsilon i}\right.$, $G_{\varepsilon i}$ and $\left.H_{\varepsilon i}\right)$ and three new 1-D left images $\left(F_{\varepsilon l i}, G_{\varepsilon l i}\right.$ and $\left.H_{\varepsilon l i}\right)$, we can obtain matrixes $\left(F_{n i}\right.$, $G_{n i}$ and $\left.H_{n i}\right)$ that only contain cross-pixels.

First, $F_{i}, G_{i}$ and $H_{i}$ are moved down by the amount of $\varepsilon_{f i}, \varepsilon_{g i}$ and $\varepsilon_{h i}$, respectively, so that $F_{\varepsilon i}, G_{\varepsilon i}$ and $H_{\varepsilon i}$ can be obtained by

$$
\left\{\begin{array}{l}
F_{\varepsilon i}=F_{i}-\varepsilon_{f i} E=\left(f_{(i, 0)}-\varepsilon_{f i}, f_{(i, 1)}-\varepsilon_{f i}, \ldots, f_{(i, M-1)}-\varepsilon_{f i}\right) \\
G_{\varepsilon i}=G_{i}-\varepsilon_{g i} E=\left(g_{(i, 0)}-\varepsilon_{g i}, g_{(i, 1)}-\varepsilon_{g i}, \ldots, g_{(i, M-1)}-\varepsilon_{g i}\right) \\
H_{\varepsilon i}=H_{i}-\varepsilon_{h i} E=\left(h_{(i, 0)}-\varepsilon_{h i}, h_{(i, 1)}-\varepsilon_{h i}, \ldots, h_{(i, M-1)}-\varepsilon_{h i}\right)
\end{array}\right.
$$

where $E$ is a 1-D matrix whose elements are all 1's, and $\varepsilon_{f i}, \varepsilon_{g i}$ and $\varepsilon_{h i}$ represent the values of reference lines for each 1-D image.

Then, three new 1-D left images, $F_{\varepsilon l i}, G_{\varepsilon l i}$ and $H_{\varepsilon l i}$, can be obtained by moving $F_{\varepsilon i}, G_{\varepsilon i}$ and $H_{\varepsilon i}$ leftwards by one pixel as

$$
\left\{\begin{array}{l}
F_{\varepsilon l i}=F_{\varepsilon i}<<1=\left(f_{(i, 1)}-\varepsilon_{f i}, \ldots, f_{(i, M-1)}-\varepsilon_{f i}, 1\right), \text { if } f_{(i, b)}=\varepsilon_{f i}, \text { then } f_{(i, b)}=f_{(i, b-1)} \\
G_{\varepsilon l i}=G_{\varepsilon i}<<1=\left(g_{(i, 1)}-\varepsilon_{g i}, \ldots, g_{(i, M-1)}-\varepsilon_{g i}, 1\right), \text { if } g_{(i, b)}=\varepsilon_{g i}, \text { then } g_{(i, b)}=g_{(i, b-1)} \\
H_{\varepsilon l i}=H_{\varepsilon i}<<1=\left(h_{(i, 1)}-\varepsilon_{h i}, \ldots, h_{(i, M-1)}-\varepsilon_{h i}, 1\right), \text { if } h_{(i, b)}=\varepsilon_{h i}, \text { then } h_{(i, b)}=h_{(i, b-1)}
\end{array}\right.
$$


where $b \epsilon(1, M-1)$. Note that in Eq. (5), their last elements are set to 1 . And from left to right, if an element value is 0 , then it is reset to take on the value of the pixel to its left. Moreover, because of the characteristics of PCTP ITO circuits, $f_{(i, 0)} \neq \varepsilon_{f i}, g_{(i, 0)} \neq \varepsilon_{g i}$ and $h_{(i, 0)} \neq \varepsilon_{h i}$.

Thus, according to Eq. (4)-(5), $F_{n i}, G_{n i}$ and $H_{n i}$ can be obtained by

$$
\left\{\begin{array}{l}
F_{n i}=F_{\varepsilon i} \times F_{\varepsilon l i}{ }^{T}=\left(f_{n(i, 0)}, f_{n(i, 1)}, \ldots, f_{n(i, M-1)}\right), \text { if } f_{n(i, j)} \leq 0 \text {, then } f_{n(i, j)}=1 \text {, else } f_{n(i, j)}=0 \\
G_{n i}=G_{\varepsilon i} \times G_{\varepsilon l i}{ }^{T}=\left(g_{n(i, 0)}, g_{n(i, 1)}, \ldots, g_{n(i, M-1)}\right), \text { if } g_{n(i, j)} \leq 0 \text {, then } g_{n(i, j)}=1 \text {, else } g_{n(i, j)}=0 \\
H_{n i}=H_{\varepsilon i} \times H_{\varepsilon l i}{ }^{T}=\left(h_{n(i, 0)}, h_{n(i, 1)}, \ldots, h_{n(i, M-1)}\right), \text { if } h_{n(i, j)} \leq 0, \text { then } h_{n(i, j)}=1 \text {, else } h_{n(i, j)}=0
\end{array}\right.
$$

In $F_{n i}, G_{n i}$ and $H_{n i}$, the pixels' values are either 0 or 1 . Those whose values are 1 are just cross-pixels. All the cross-pixels in the 1-D images constitute three new 1-D matrixes for defect detection: $F_{c i}=\left(f_{n(i, k l)}, f_{n(i, k 2)}, \ldots, f_{n(i, k)}\right), G_{c i}=\left(g_{n(i, q 1)}, g_{n(i, q 2)}, \ldots, g_{n(i, q)}\right)$ and $H_{c i}=\left(h_{n(i, z l)}\right.$, $\left.h_{n(i, z 2)}, \ldots, h_{n(i, z)}\right)$, where $K=(k 1, k 2, \ldots, k), Q=(q 1, q 2, \ldots, q)$ and $Z=(z 1, z 2, \ldots, z)$ represent the coordinates of cross-pixels in $F_{i}, G_{i}$ and $H_{i}$, respectively.

Since we only need to extract one template image, any one of the three reference images can be used as the target reference image. Let's choose $\mathrm{P}_{1}$. Then $F_{i}$ is the target 1$\mathrm{D}$ image. The rest two reference images, $\mathrm{P}_{2}$ and $\mathrm{P}_{3}$, are set as the supporting reference images, and $G_{i}$ and $H_{i}$, are set as the supporting1-D images. In order to locate the defects in $F_{i}$, the active judgment matrix, $J_{(i, K)}$, and the reference judgment matrix, $R_{(i, Q)}$, are calculated first by

$$
\begin{gathered}
J_{(i, K)}=\left[\begin{array}{lll}
f_{n(i, K)}-h_{n(i, K)} & f_{n(i, K)}-h_{n(i, K+1)} & f_{n(i, K)}-h_{n(i, K-1)} \\
f_{n(i, K)}-g_{n(i, K)} & f_{n(i, K)}-g_{n(i, K+1)} & f_{n(i, K)}-g_{n(i, K-1)}
\end{array}\right]=\left[\begin{array}{lll}
c_{1} & c_{2} & c_{3} \\
c_{4} & c_{5} & c_{6}
\end{array}\right] \\
R_{(i, Q)}=\left[\begin{array}{lll}
g_{n(i, Q)}-f_{n(i, Q)} & g_{n(i, Q)}-f_{n(i, Q+1)} & g_{n(i, Q)}-f_{n(i, Q-1)} \\
g_{n(i, Q)}-h_{n(i, Q)} & g_{n(i, Q)}-h_{n(i, Q+1)} & g_{n(i, Q)}-h_{n(i, Q-1)}
\end{array}\right]=\left[\begin{array}{lll}
r_{1} & r_{2} & r_{3} \\
r_{4} & r_{5} & r_{6}
\end{array}\right]
\end{gathered}
$$

In Eq. (7)-(8), $c_{1}$ and $c_{4}$ are the differences between the cross-pixel whose coordinate is $K$ in $F_{n i}$ and the pixel of the same location in $H_{n i}$ and $G_{n i}$, respectively. $c_{2}$ and $c_{5}$ are the differences between the cross-pixel whose coordinate is $K$ in $F_{n i}$ and the pixel whose coordinate is $K+1$ in $H_{n i}$ and $G_{n i}$, respectively. $c_{3}$ and $c_{6}$ are the differences between the cross-pixel whose coordinate is $K$ in $F_{n i}$ and the pixel whose coordinate is $K-1$ in $H_{n i}$ and $G_{n i}$, respectively. $r_{l}$ and $r_{4}$ are the differences between the cross-pixel whose coordinate is $Q$ in $G_{n i}$ and the pixel of the same location in $F_{n i}$ and $H_{n i}$, respectively. $r_{2}$ and $r_{5}$ are the differences between the cross-pixel whose coordinate is $Q$ in $G_{n i}$ and the pixel whose coordinate is $Q+1$ in $F_{n i}$ and $H_{n i}$, respectively. $r_{3}$ and $r_{6}$ are the differences between the cross-pixel whose coordinate is $Q$ in $G_{n i}$ and the pixel whose coordinate is $Q-1$ in $F_{n i}$ and $H_{n i}$, respectively. According to the judgment matrixes above, the procedure of defect detection is as follows.

Step 1.

Based on $J_{(i, K)}$, the defect-cross-pixels in the target 1-D image, $F_{i}$, can be located by

$\left\{\begin{array}{l}V_{1}=c_{1} \& c_{2} \& c_{3}, V_{2}=c_{4} \& c_{5} \& c_{6} ; \\ \text { if } V_{1}=0, V_{2}=0, \text { no defect } \\ \text { if } V_{1}=1, V_{2}=1, f_{(i, K)} \text { is a defect } \text { - cross - pixel }\end{array}\right.$

Step 2. 
Based on $R_{(i, Q)}$, for the cross-pixels in $G_{i}$ whose coordinates do not overlap with those in $F_{i}$, i.e., $Q \notin(K-1, K+1)$, the defect-cross-pixels can be obtained by

$\left\{\begin{array}{l}V_{3}=r_{a 1} \& r_{a 2} \& r_{a 3}, V_{4}=r_{a 4} \& r_{a 5} \& r_{a 6} ; \\ \text { if } V_{3}=1, V_{4}=0, f_{(i, Q)} \text { is a defect-cross-pixel }\end{array}\right.$

The above detection procedure can only find pixels located at the two ends of the defect area, hence the name defect-cross-pixels. Moreover, as can be seen in the 1-D images of PCTP ITO circuits, defect-cross-pixels always appear in pairs. Suppose the number of defect cross-pixels in $F_{i}$ is $e$, and they can be represented by $F_{d i}=\left(f_{(i, c l)}\right.$, $\left.f_{n(i, c 2)}, \ldots, f_{n(i, c e)}\right)$, where $e$ is an even integer. Every defect in $F_{i}$ can then be expressed as an interval consisting of every two elements from left to right in $F_{d i}$. That is, every defect can be represented by an interval $\left(f_{(i, c s)}, f_{n(i, c(s+1))}\right)$, where $s=(1,2, \ldots, e)$.

For example, Figure 5, illustrates $F_{\varepsilon 28}, G_{\varepsilon 28}$ and $H_{\varepsilon 28}$ that are extracted from $\mathrm{P}_{1}, \mathrm{P}_{2}$ and $\mathrm{P}_{3}$ in Figure 3 , respectively. For every cross-pixel in $F_{\varepsilon 28}$, by calculating $J_{(28, K)}$ we get $V_{1}=0$ and $V_{2}=0$. For the cross-pixels in $G_{\varepsilon 28}$ whose coordinates do not overlap with those in $F_{\varepsilon 28}$, i.e., $Q=(251,256)$, we can calculate $R_{(28, Q)}$ and get $V_{3}=1$ and $V_{4}=0$. Thus, $f_{(28,251)}$ and $f_{(28,256)}$ are two defect-cross-pixels, and the interval, $\left(f_{(28,251)}, f_{(28,256)}\right)$, represents a defect that exists in $F_{28}$.

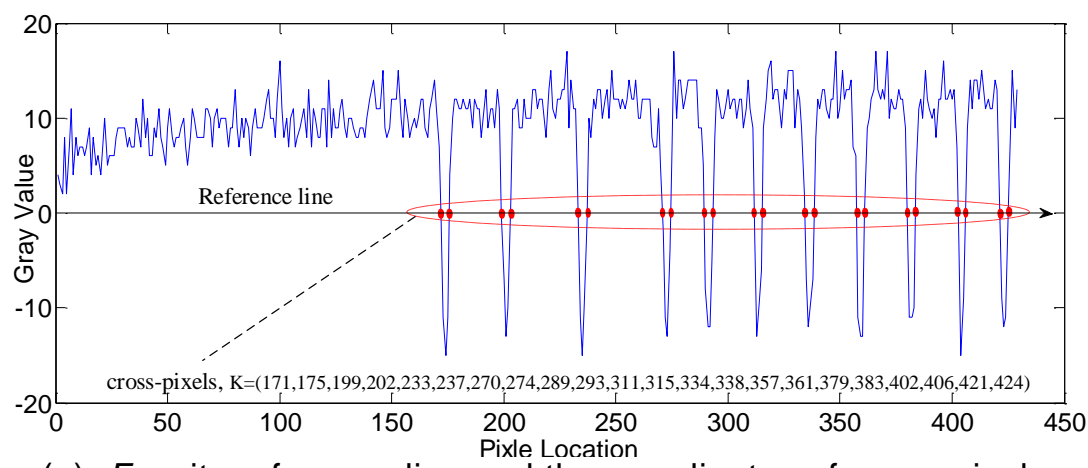

(a). $F_{\varepsilon 28}$, its reference line and the coordinates of cross-pixels

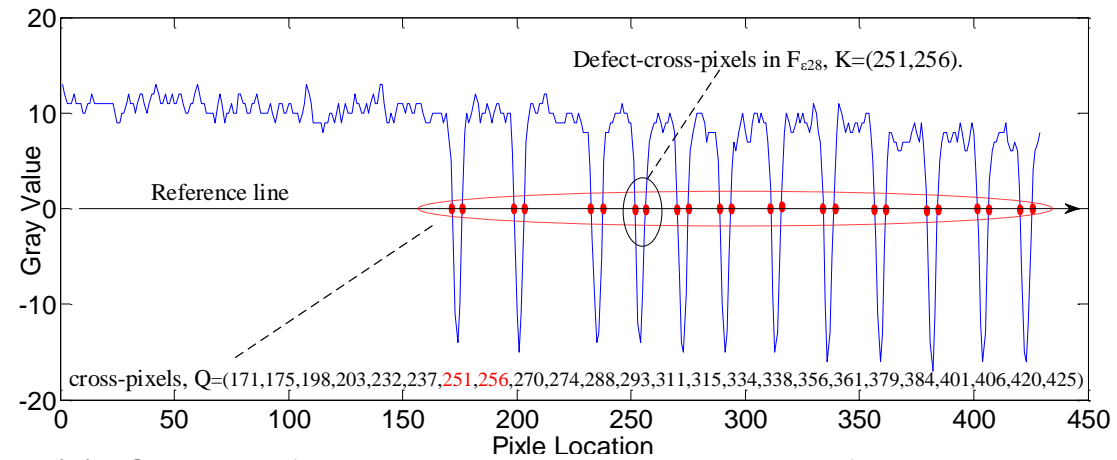

(b). $G_{\varepsilon 28}$, its reference line and the coordinates of cross-pixels 


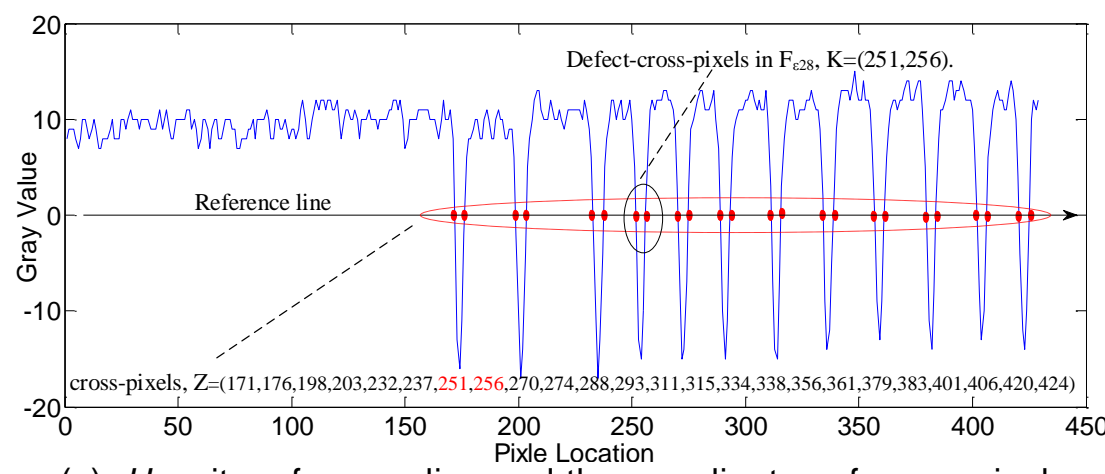

(c). $H_{\varepsilon 28}$, its reference line and the coordinates of cross-pixels

Figure 5. Cross-Pixels and Defect-Cross-Pixels

2.1.4. Replacing Defects in the Target Reference Image: After defect intervals are extracted in the target 1-D image $F_{i}$, they can be replaced by the faultless pixels located in the same position of either $G_{i}$ or $H_{i}$. However, considering the possibilities of image deformation and uneven grayscale distribution, this paper uses Pearson correlation coefficient to pick from $G_{i}$ and $H_{i}$ faultless pixels that are highly correlated with pixels near the defect in $F_{i}$.

Suppose that interval $\left(f_{(i, k 3)}, f_{(i, k 4)}\right)$ represents a defect in $F_{i} . f_{(i, k 2)}$ is a cross-pixel before $f_{(i, k 3)}$, and $f_{(i, k 5)}$ is a cross-pixel after $f_{(i, k 4)}$. Then, the pixel intervals that need to be compared are $\left(f_{(i, k 2+1)}, f_{(i, k 3-1)}\right)$ versus $\left(f_{(i, k 4+1)}, f_{(i, k 5-1)}\right),\left(g_{(i, k 2+1)}, g_{(i, k 3-1)}\right)$ versus $\left(g_{(i, k 4+1)}, g_{(i, k 5-1)}\right)$ and $\left(h_{(i, k 2+1)}, h_{(i, k 3-1)}\right)$ versus $\left(h_{(i, k 4+1)}, h_{(i, k 5-1)}\right)$. Re-write as the following new 1-D matrixes, $F_{r e}, G_{r e}$ and $H_{r e}$ :

$$
\left\{\begin{array}{l}
F_{r e}=\left(f_{\left(i, k_{2}+1\right)}, \ldots, f_{\left(i, k_{3}-1\right)}, f_{\left(i, k_{4}+1\right)}, \ldots, f_{\left(i, k_{5}-1\right)}\right) \\
G_{r e}=\left(g_{\left(i, k_{2}+1\right)}, \ldots, g_{\left(i, k_{3}-1\right)}, g_{\left(i, k_{4}+1\right)}, \ldots, g_{\left(i, k_{5}-1\right)}\right) \\
H_{r e}=\left(h_{\left(i, k_{2}+1\right)}, \ldots, h_{\left(i, k_{3}-1\right)}, h_{\left(i, k_{4}+1\right)}, \ldots, h_{\left(i, k_{5}-1\right)}\right)
\end{array}\right.
$$

Note that since the gray values of pixels in the compared intervals are approximately distributed as the combination of two Normal distributions, and the Pearson method is only applicable to one Normal distribution, we need to segment the intervals by thresholds first. The threshold values, $\varepsilon_{1}, \varepsilon_{2}$ and $\varepsilon_{3}$, can also be obtained by automatic threshold processing. Then the matrixes, $F_{r e l}, F_{r e h}, G_{r e l}, G_{r e h}, H_{r e l}$, and $H_{r e h}$, after threshold segmentation are

$\left\{\begin{array}{l}\text { if }\left(f_{\left(i, k_{2}+1\right)}, \ldots, f_{\left(i, k_{3}-1\right)}, f_{\left(i, k_{4}+1\right)}, \ldots, f_{\left(i, k_{5}-1\right)}\right)<\varepsilon_{1}, f_{(i, y) l} \in F_{r e l} \\ \text { if }\left(f_{\left(i, k_{2}+1\right)}, \ldots, f_{\left(i, k_{3}-1\right)}, f_{\left(i, k_{4}+1\right)}, \ldots, f_{\left(i, k_{5}-1\right)}\right)>\varepsilon_{1}, f_{(i, y) h} \in F_{r e h}\end{array}\right.$

$\left\{\begin{array}{l}\text { if }\left(g_{\left(i, k_{2}+1\right)}, \ldots, g_{\left(i, k_{3}-1\right)}, g_{\left(i, k_{4}+1\right)}, \ldots, g_{\left(i, k_{5}-1\right)}\right)<\varepsilon_{2}, g_{(i, y) l} \in G_{r e l} \\ \text { if }\left(g_{\left(i, k_{2}+1\right)}, \ldots, g_{\left(i, k_{3}-1\right)}, g_{\left(i, k_{4}+1\right)}, \ldots, g_{\left(i, k_{5}-1\right)}\right)>\varepsilon_{2}, g_{(i, y) h} \in G_{r e h}\end{array}\right.$

$\left\{\begin{array}{l}\text { if }\left(h_{\left(i, k_{2}+1\right)}, \ldots, h_{\left(i, k_{3}-1\right)}, h_{\left(i, k_{4}+1\right)}, \ldots, h_{\left(i, k_{5}-1\right)}\right)<\varepsilon_{3}, h_{(i, y)} \in H_{r e l} \\ \text { if }\left(h_{\left(i, k_{2}+1\right)}, \ldots, h_{\left(i, k_{3}-1\right)}, h_{\left(i, k_{4}+1\right)}, \ldots, h_{\left(i, k_{5}-1\right)}\right)>\varepsilon_{3}, h_{(i, y)} \in H_{r e h}\end{array}\right.$

Let $r_{i-}, r_{i+}, o_{i-}$ and $o_{i+}$ represent the Pearson correlation coefficients between $F_{r e l}$ and $G_{r e l}, F_{r e h}$ and $G_{r e h}, F_{r e l}$ and $H_{r e l}, F_{r e h}$ and $H_{r e h}$, respectively. They can be obtained by 


$$
\begin{gathered}
r_{i-}=\frac{\left\langle f_{(i, y) l}-\bar{F}_{r e l}, g_{(i, y) l}-\bar{G}_{r e l}\right\rangle}{\left\|f_{(i, y) l}-\bar{F}_{r e l}\right\|\left\|g_{(i, y) l}-\bar{G}_{r e l}\right\|}=\frac{\sum_{y}\left(f_{(i, y) l}-\bar{F}_{r e l}\right)\left(g_{(i, y) l}-\bar{G}_{r e l}\right)}{\sqrt{\sum_{y}\left(f_{(i, y) l}-\bar{F}_{r e l}\right)^{2}} \sqrt{\sum_{y}\left(g_{(i, y) l}-\bar{G}_{r e l}\right)^{2}}} \\
r_{i+}=\frac{\left\langle f_{(i, y) h}-\bar{F}_{r e h}, g_{(i, y) h}-\bar{G}_{r e h}>\right.}{\left\|f_{(i, y) h}-\bar{F}_{r e h}\left|\left\|\mid g_{(i, y) h}-\bar{G}_{r e h}\right\|\right.\right.}=\frac{\sum_{y}\left(f_{(i, y) h}-\bar{F}_{r e h}\right)\left(g_{(i, y) h}-\bar{G}_{r e h}\right)}{\sqrt{\sum_{y}\left(f_{(i, y) h}-\bar{F}_{r e h}\right)^{2}} \sqrt{\sum_{y}\left(g_{(i, y) h}-\bar{G}_{r e h}\right)^{2}}} \\
o_{i-}=\frac{\left\langle f_{(i, y) l}-\bar{F}_{r e l}, h_{(i, y) l}-\bar{H}_{r e l}>\right.}{\left\|f_{(i, y) l}-\bar{F}_{r e l}\left|\left\|\mid h_{(i, y) l}-\bar{H}_{r e l}\right\|\right.\right.}=\frac{\sum_{y}\left(f_{(i, y) l}-\bar{F}_{r e l}\right)\left(h_{(i, y) l}-\bar{H}_{r e l}\right)}{\sqrt{\sum_{y}\left(f_{(i, y) l}-\bar{F}_{r e l}\right)^{2}} \sqrt{\sum_{y}\left(h_{(i, y) l}-\bar{H}_{r e l}\right)^{2}}} \\
o_{i+}=\frac{\left\langle f_{(i, y) h}-\bar{F}_{r e h}, h_{(i, y) h}-\bar{H}_{r e h}>\right.}{\left\|f_{(i, y) h}-\bar{F}_{r e h}\right\|\left\|h_{(i, y) h}-\bar{H}_{r e h}\right\|}=\frac{\sum_{y}\left(f_{(i, y) h}-\bar{F}_{r e h}\right)\left(h_{(i, y) h}-\bar{H}_{r e h}\right)}{\sqrt{\sum_{y}\left(f_{(i, y) h}-\bar{F}_{r e h}\right)^{2}} \sqrt{\sum_{y}\left(h_{(i, y) h}-\bar{H}_{r e h}\right)^{2}}}
\end{gathered}
$$

where $\bar{F}_{r e l}, \bar{F}_{r e h}, \bar{G}_{r e l}, \bar{G}_{r e h}, \bar{H}_{r e l}$ and $\bar{H}_{r e h}$ are the mean values of $F_{r e l}, F_{r e h}, G_{r e l}, G_{r e h}$, $H_{r e l}$ and $H_{r e h}$, respectively. And $f_{(i, y) l}, f_{(i, y) h}, g_{(i, y) l}, g_{(i, y) h}, h_{(i, y) l}$ and $h_{(i, y) h}$ represent the pixels in $F_{r e l}, F_{r e h}, G_{r e l}, G_{r e h}, H_{r e l}$ and $H_{r e h}$, respectively. Thus, the replacement can be implemented by

$$
\left\{\begin{array}{l}
\text { if } r_{i-}+r_{i+}>o_{i-}+o_{i+}, \text { then }\left(f_{\left(i, k_{2}\right)}, \ldots, f_{\left(i, k_{3}\right)}\right)^{T}=\left(g_{\left(i, k_{2}\right)}, \ldots, g_{\left(i, k_{3}\right)}\right)^{T} \\
\text { if } r_{i-}+r_{i+}<o_{i-}+o_{i+}, \text { then }\left(f_{\left(i, k_{2}\right)}, \ldots, f_{\left(i, k_{3}\right)}\right)^{T}=\left(h_{\left(i, k_{2}\right)}, \ldots, h_{\left(i, k_{3}\right)}\right)^{T}
\end{array}\right.
$$

A faultless template image can be extracted by using Eq. (4)-(19) repeatedly setting $i=i+1(0 \leq i \leq N)$ repeatedly.

\subsection{Detecting Defects Using the Template Image}

Defects in PCTP ITO circuits can be quickly detected by using the faultless template obtained from the above section. Let $\mathrm{P}$ be a sensed image, and $\mathrm{T}$ be the faultless template image. $P_{i}=\left(p_{(i, 0)}, p_{(i, 1)}, \ldots, p_{(i, M-1)}\right)$, the sensed 1-D image, and $T_{i}=\left(t_{(i, 0)}, t_{(i, 1)}, \ldots, t_{(i, M-1)}\right)$, the template 1-D image, are rows extracted from $\mathrm{P}$ and $\mathrm{T}$, respectively. Base on the same principle as Eq. (4)-(5), two new 1-D images, $P_{\varepsilon i}$ and $T_{\varepsilon i}$, and two 1-D left images, $P_{\varepsilon l i}$ and $T_{\varepsilon l i}$, can be obtained by

$$
\begin{aligned}
& \left\{\begin{array}{l}
T_{\varepsilon i}=T_{i}-\varepsilon_{t i} E=\left(t_{(i, 0)}-\varepsilon_{t i}, t_{(i, 1)}-\varepsilon_{t i}, \ldots, t_{(i, M-1)}-\varepsilon_{t i}\right) \\
P_{\varepsilon i}=P_{i}-\varepsilon_{p i} E=\left(p_{(i, 0)}-\varepsilon_{p i}, p_{(i, 1)}-\varepsilon_{p i}, \ldots, p_{(i, M-1)}-\varepsilon_{p i}\right)
\end{array}\right. \\
& \left\{\begin{array}{l}
T_{\varepsilon l i}=T_{\varepsilon i}<<1=\left(t_{(i, 1)}-\varepsilon_{t i}, \ldots, t_{(i, M-1)}-\varepsilon_{t i}, 1\right), \text { if } t_{(i, j)}=\varepsilon_{t i}, \text { then } t_{(i, j)}=t_{(i, j-1)} \\
P_{\varepsilon l i}=P_{\varepsilon i}<<1=\left(p_{(i, 1)}-\varepsilon_{p i}, \ldots, p_{(i, M-1)}-\varepsilon_{p i}, 1\right), \text { if } p_{(i, j)}=\varepsilon_{p i}, \text { then } p_{(i, j)}=p_{(i, j-1)}
\end{array}\right.
\end{aligned}
$$

where $\varepsilon_{t i}$ and $\varepsilon_{p i}$ are the values of reference lines obtained by automatic threshold processing. In order to extract cross-pixels, $T_{n i}$ and $P_{n i}$ can be calculated by 
$\left\{\begin{array}{l}T_{n i}=T_{\varepsilon i} \times T_{\varepsilon l i}^{T}=\left(t_{n(i, 0)}, t_{n(i, 1)}, \ldots, t_{n(i, M-1)}\right), \text { if } t_{n(i, j)} \leq 0 \text {, then } t_{n(i, j)}=1 \text {, else } t_{n(i, j)}=0 \\ P_{n i}=P_{\varepsilon i} \times P_{\varepsilon l i}{ }^{T}=\left(p_{n(i, 0)}, p_{n(i, 1)}, \ldots, p_{n(i, M-1)}\right), \text { if } p_{n(i, j)} \leq 0 \text {, then } p_{n(i, j)}=1 \text {, else } p_{n(i, j)}=0\end{array}\right.$

In $T_{n i}$ and $P_{n i}$, all the cross-pixels can be represented by $P_{c i}=\left(p_{(i, d l)}, p_{(i, d 2)}, \ldots, p_{(i, d)}\right)$ and $T_{c i}=\left(t_{(i, w 1}, t_{(i, w 2)}, \ldots, t_{(i, w)}\right)$. Then, whether the sensed 1-D image contains defects can be easily determined by comparing the number of cross-pixels between $P_{c i}$ and $T_{c i}$ :

$\left\{\begin{array}{l}\text { if } C_{p}=C_{t}, \text { no defect in } P_{i} \\ \text { if } C_{p} \neq C_{t} \text {, the defect in } P_{i}\end{array}\right.$

where $C_{p}$ and $C_{t}$ represent the number of cross-pixels in $P_{i}$ and $T_{i}$, respectively. If $C_{p} \neq$ $C_{t}$, defects exist in $P_{i}$, and the location of the defect-cross-pixels can be obtained by calculating the judgment matrix, $J_{(i, D)}$, as

$J_{(i, D)}=\left[t_{n(i, W)}-p_{n(i, D)} \quad t_{n(i, W)}-p_{n(i, D+1)} \quad t_{n(i, W)}-p_{n(i, D-1)}\right]=\left[\begin{array}{lll}c_{t 1} & c_{t 2} & c_{t 3}\end{array}\right]$

$\left\{\begin{array}{l}V_{t 1}=c_{t 1} \& c_{t 2} \& c_{t 3} \\ \text { if } V_{t 1}=0, \text { no defect } \\ \text { if } V_{t 1} \neq 0, p_{(i, D)} \text { is a defect cross - pixel }\end{array}\right.$

where $W=\left(w_{1}, w_{2}, \ldots, w\right)$ and $D=\left(d_{1}, d_{2}, \ldots, d\right)$ respectively represent the coordinates of all cross-pixels in $T_{c i}$ and $P_{c i}$. Suppose there are x defect-cross-pixels in $P_{i}$, where $x$ is an even integer, and $P_{d i}=\left(p_{(i, y l)}, p_{n(i, y 2)}, \ldots, p_{n(i, y x)}\right)$ represents the defect-cross-pixels in $P_{i}$. Then, every defect can be represented by an interval $\left(p_{(i, y u)}, p_{(i, y(u+1))}\right)$, where $u=(1,2, \ldots, e)$. As we move onto $i=i+1(0 \leq i \leq N)$, all defects in the test image can be identified by repeating Eq. (20)-(25), and can be displayed in a binary image.

Since our defect detection algorithm only requires comparing the number of crosspixels between test and template 1-D images, it is computationally simple and hence can achieve fast and accurate real-time inspection.

\section{Experiment Results}

In order to evaluate the efficiency and effectiveness of the proposed 1-D image processing method, several ITO circuit images with a variety of defects including scratches, pinholes, particles, shut circuits and open circuits are inspected. The sensed images are of size $209 \times 429$ pixels. That is, a sensed image is comprised of 209 scan lines, each of the length 429 pixels. Figure $3(\mathrm{a}-\mathrm{c})$ show three defective 2-D sensed images, $\mathrm{P}_{1}$, $\mathrm{P}_{2}$ and $\mathrm{P}_{3}$, under illumination of 1500 lux at a fine resolution of 100 pixels $/ \mathrm{mm}$. Suppose defects that are larger than 2 pixels in 1-D images need to be detected.

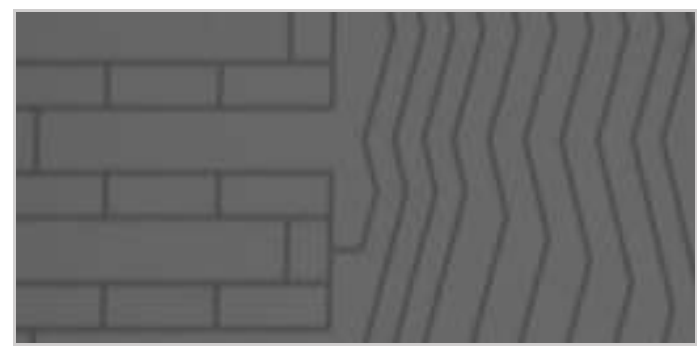

(a) The faultless template image

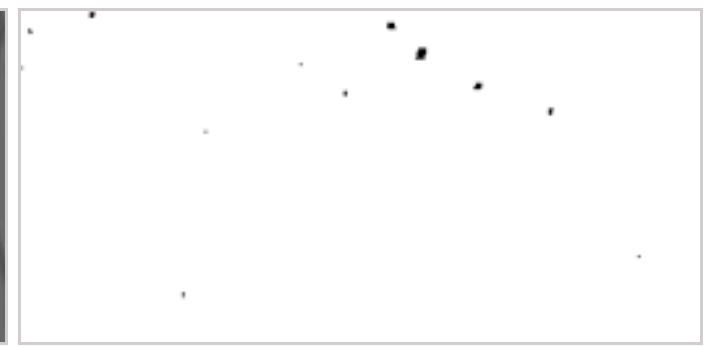

(b) Defects in $P_{1}$ 


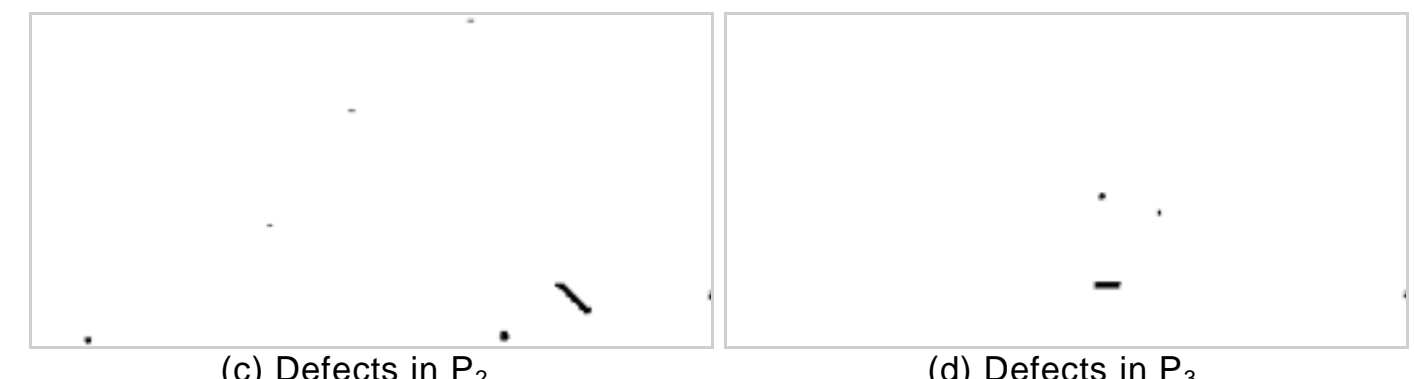

Figure 6. The Faultless Template Image and Defects in Three Sensed Images

Figure $6(\mathrm{a}-\mathrm{d})$, present the template extraction and detection results for the three sensed images. All defects are well segmented and accurately identified. On a computer with a memory of $4 \mathrm{G}$ and $\mathrm{CPU}$ of $2.49 \mathrm{GHz}$, the average time to extract a template images is 0.42 seconds, and the average time to detect all defects in a sensed image is 0.23 seconds. The experiment results confirm that the proposed method is fast and accurate.

In order to further demonstrate the robustness of the proposed algorithm when illumination condition changes as is often the case in real inspection, five versions of defective images under illuminations of 500, 1000, 1500, 2000 and 2500 lux are used as test images in Figure 7 (a1-e1). Figure 6 (a), is taken as the template image. Note that Figure 7 (a1, e1), are two highly underexposed and overexposed images for inspection. The detection results are presented in Figure 7 (a2-e2), as binary images.

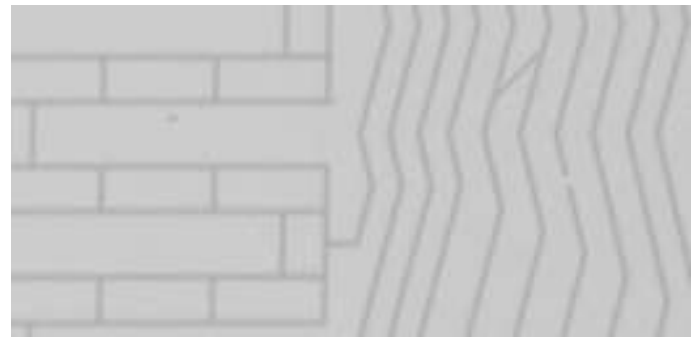

(a1) A image under 2500 lux

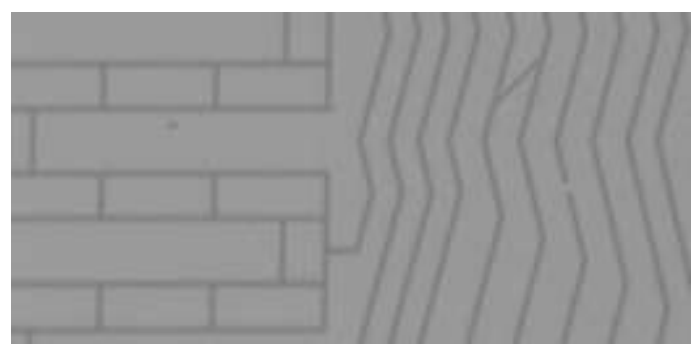

(b1) A image under 2000 lux

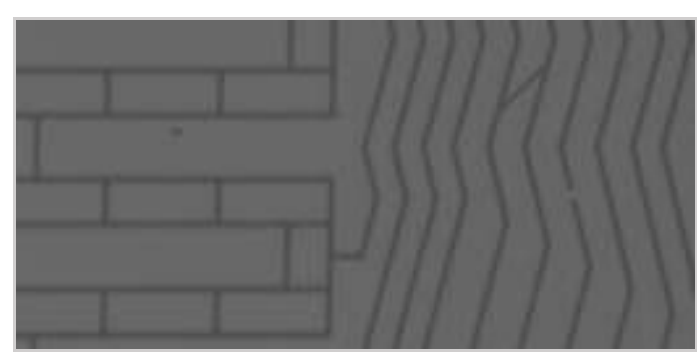

(c1) A image under 1500 lux

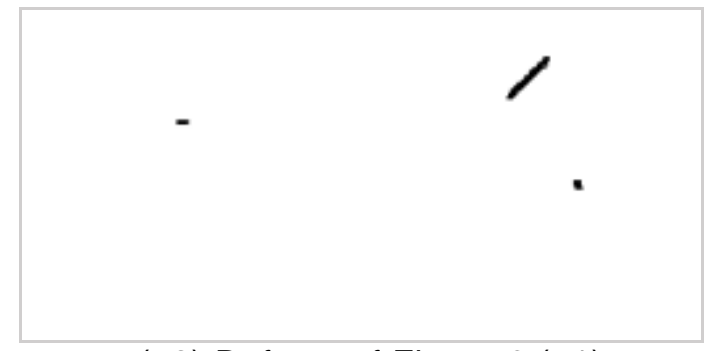

(a2) Defects of Figure 6 (a1)

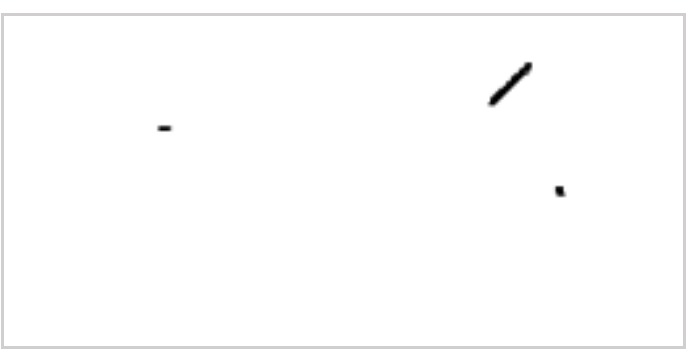

(b2) Defects of Figure 6 (b1)

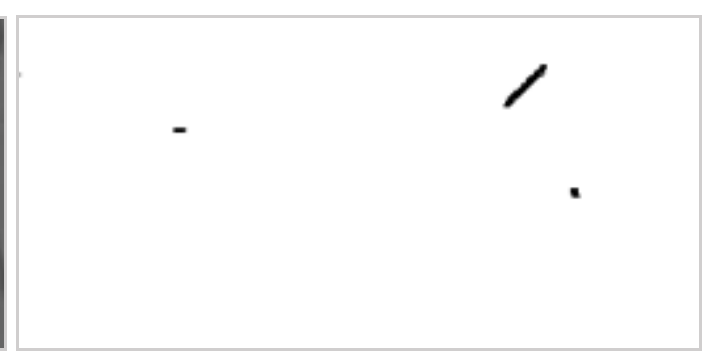

(c2) Defects of Figure 6 (c1) 


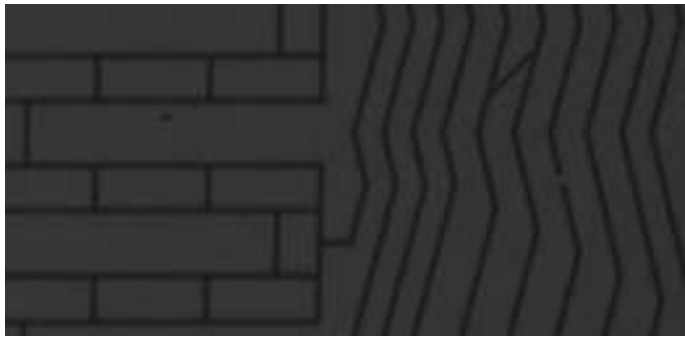

(d1) A image under 1000 lux

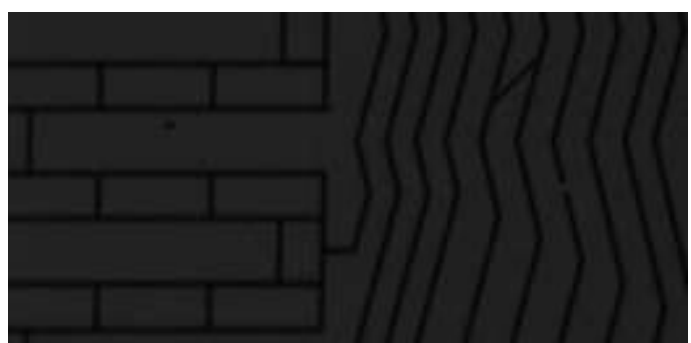

(e1) A image under 500 lux

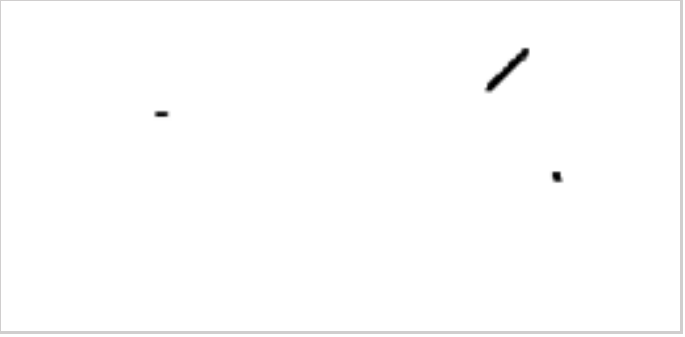

(d2) Defects of Figure 6 (d1)

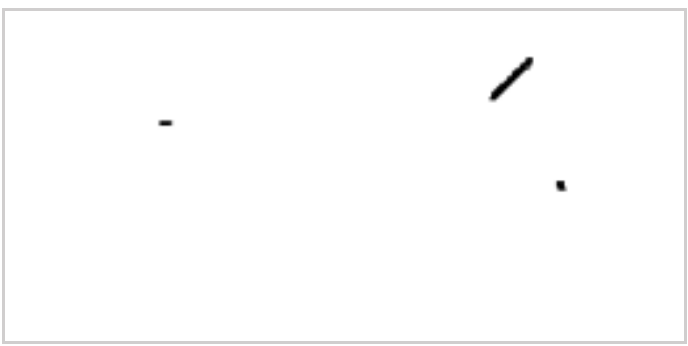

(e2) Defects of Figure 6 (e1)

Figure 7. Detection Results under Different Brightness

Note that the detection result in Figure 7(c2), is obtained under the same illumination condition as the template image. Thus, other detection results in Figure 7(a2-e2), are subtracted from Figure 7(c2), in order to evaluate detection accuracy under different brightness. The subtraction results show that areas with different pixels are less than $1 \%$ of total area. Thus, our method can detect defects under different illumination conditions, even the overly dark or bright ones. It can be reliably used in real inspection.

\section{Conclusions}

In this paper, a new defect automatic visual inspection method based on 1-D images is presented to inspect the surfaces of PCTP ITO circuits. The main advantages of the proposed method include its succinct mathematical description, simple algorithm flow and insensitivity to image deformation. Moreover, it remains highly effective when illumination condition changes in a wide range from 500 to 2500 lux. We conducted experiments to confirm that the proposed method is fast, accurate and robust. Therefore, it can be reliably used in real inspection.

\section{Acknowledgments}

The authors thank the Science and Technology Department of Guangdong, China, for the financial support through Grant 2013B010402003.

\section{References}

[1] A. Holzinger, "Finger Instead of Mouse: Touch Screens As a Means of Enhancing Universal Access", $7^{\text {th }}$ ERCIM International Workshop on User Interfaces, Paris, France, (2002) October 24-25.

[2] D. S. Leem, T. Lee and T. Y. Seong, "Enhancement of the Light Output of $\mathrm{G}_{\mathrm{a}} \mathrm{N}$-based Light-emitting Diodes with Surface-patterned ITO Electrodes by Maskless Wet-etching", Solid-state Electronics, vol. 51, no. 5, (2007), pp. 793-796.

[3] J. Y. Park, H. S. Kim and D. H. Lee, "A Study on the Etch Characteristics of ITO Thin Film Using Inductively Coupled Plasmas", Surface and Coatings Technology, vol. 131, no. 1, (2000), pp. 247-251.

[4] M. F. Chen, Y. P. Chen and W. T. Hsiao, "Laser Direct Write Patterning Technique of Indium Tin Oxide Film", Thin Solid Films, vol. 515, no. 24, (2007), pp. 8515-8518.

[5] C. H. Chan, Y. T. Zou and T. K. Liu, "The Development of an Inspection System for Indium Tin Oxide Circuits", Measurement Science and Technology, vol. 23, no. 8, (2012), pp. 085902. 
[6] J. S. Gao, K. Wang and J. J. Sun, "Study on the Technology of Ultrasonic Imaging Detection Based on Phase Array", International Journal of Signal Processing, Image Processing and Pattern Recognition, vol. 6, no. 5, (2013), pp.71-78.

[7] B. Y. Chen, Y. Q. Lan, J. Y. Liu, Z. H. Li and X. Y. Yu, "Voice Activity detection algorithm based on improved radial basis function neural network", International Journal of Signal Processing, Image Processing and Pattern Recognition, vol. 7, no. 5, (2014), pp.187-196.

[8] S. M. Ramesh, B. Gomathy and T. Sundararajan, "Detection of Defects on Steel Surface for Using Image Segmentation Techniques", International Journal of Signal Processing, Image Processing and Pattern Recognition, vol. 7, no. 5, (2014), pp.323-332.

[9] A. Hou, W. Zhou and G. Cui, "Study on Defect Detection of IC Wafer Based on Morphology", Photonics Asia 2007, Beijing, China, (2007) November 11-15.

[10] D. M. Tsai and C. Y. Hung, "Automatic Defect Inspection of Patterned Thin Film Transistor-liquid Crystal Display (TFT-LCD) Panels Using One-dimensional Fourier Reconstruction and Wavelet Decomposition”, International Journal of Production Research, vol. 43, no. 21, (2005), pp. 4589-4607.

[11] D. M. Tsai and S. C. Lai, "Defect Detection in Periodically Patterned Surfaces Using Independent Component Analysis", Pattern Recognition, vol. 41, no. 9, (2008), pp.2812-2832.

[12] H. X. Liu, W. Zhou, Q. W. Kuang, L. Cao and B. Gao, "Defect Detection of IC Wafer Based on Spectral Subtraction", IEEE Transactions on Semiconductor Manufacturing, vol. 23, no. 1, (2010), pp.141-147.

[13] H. X. Liu, W. Zhou, Q. W. Kuang, L. Cao and B. Gao, "Defect Detection of IC Wafer Based on TwoDimension Wavelet Transform", Microelectronics Journal, vol. 41, no. 2, (2010), pp.171-177.

[14] J. Y. Song, H. Y. Park, H. J. Kim and Y. W. Jung, "Development of Defect Inspection System for PDP ITO Patterned Glass", International Journal of Precision Engineering \& Manufacturing, vol. 7, no. 3, (2006), pp.18-23.

[15] Z. Q. Lu and Y. L. Peng, "A Defect Inspection Algorithm for LCD Touch Screen”, ICISE2009, Nanjing, China, (2009) December 26-28.

[16] R. C. Gonzalez, R. E. Woods and S. L. Faddins, Editor, "Digital Image Processing Using MATLAB, 2nd edition”, Prentice Hall, Upper Saddle River, (2009).

\section{Authors}

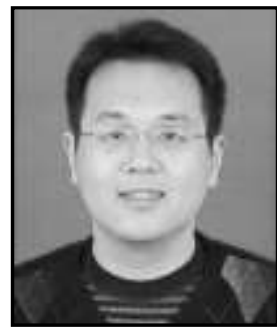

Changcheng Jiang, received his M.Sc. and Ph.D. degree in Measurement Techniques \& Measuring Instruments and Mechanical Manufacturing and Automation from South China University of Technology in 2012 and 2016, respectively. His research interests are machine vision and AOI equipment.

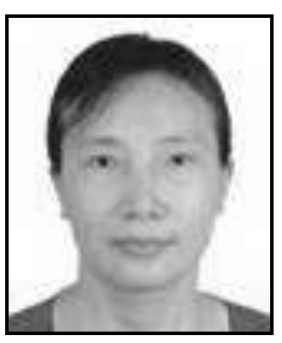

Yanming Quan, received her M.Sc and Ph.D degree both in Mechanical Manufacturing and Automation from South China University of Technology in 1993 and 1997. Currently, she is a Professor at the School of Mechanical and Automotive Engineering, South China University of Technology. Her research interests include advanced manufacturing, machine vision and measurement techniques.

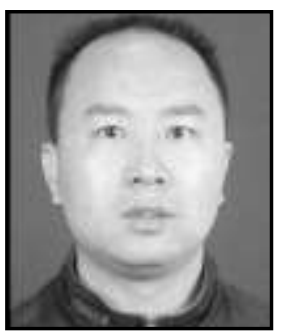

Xingui Lin, received his M.Sc in Mechanical Manufacturing and Automation from Tongji University in 2004. Currently, he is an Associate Professor at the School of Electro Mechanic Engineering, Guangzhou Panyu Polytechnic. His research interests include advanced manufacturing and machine vision. 